\title{
8. REGULATIONS AND ADVISORIES
}

International guidelines for ammonia were not located. National and state regulations and guidelines pertinent to human exposure to ammonia are summarized in Table 8-1.

ATSDR has derived an acute-duration inhalation MRL of $1.7 \mathrm{ppm}$ for ammonia based on a minimal LOAEL of $50 \mathrm{ppm}$ for eye, nose, and throat irritation in a study with volunteers (Verberk et al. 1977). No NOAEL was identified in that study. An uncertainty factor of 30 (3 for the use of a minimal LOAEL and 10 to protect sensitive individuals) was applied to the LOAEL.

ATSDR has derived a chronic-duration inhalation MRL of $0.1 \mathrm{ppm}$ for ammonia based on a NOAEL of $9.2 \mathrm{ppm}$ for sense of smell, prevalence of respiratory symptoms (cough, bronchitis, wheeze, dyspnea, etc.), eye and throat irritation, and pulmonary function parameters in workers exposed for approximately 12 years in a soda ash plant (Holness et al. 1989). No LOAEL was defined in that study. The NOAEL was duration-adjusted, and divided by an uncertainty factor of 10 to protect sensitive individuals. A modifying factor of 3 was added for the lack of reproductive and developmental studies. This MRL supersedes the previous chronic inhalation MRL of $0.3 \mathrm{ppm}$ derived in the $2002 \mathrm{draft}$ for public comment version of this profile.

EPA derived an inhalation reference concentration (RfC) of $1 \mathrm{E}-1 \mathrm{mg} / \mathrm{m}^{3}(0.14 \mathrm{ppm})$ for ammonia based on a NOAEL of $6.4 \mathrm{mg} / \mathrm{m}^{3}$ (9.2 ppm) defined in the Holness et al. (1989) study (IRIS 2004). EPA used an uncertainty factor of 30 (10 to protect sensitive individuals and 3 for data base deficiencies).

Ammonia has not undergone a complete evaluation under EPA's IRIS program for evidence of human carcinogenic potential.

Ammonium ion is regulated by the Clean Water Effluent Guidelines for the following industrial point sources: ferroalloy manufacturing; fertilizer manufacturing; glass manufacturing; inorganic chemicals; iron and steelmaking; landfills; nonferrous metals manufacturing; nonferrous metals forming and metal powder; paper and paperboard; petroleum refining; pharmaceutical manufacturing; pulp, meat products; and transportation equipment cleaning (EPA 2002j). 
The FDA (1973) determined that concentrations of ammonia and ammonium compounds normally present in food do not suggest a health risk; ammonia and ammonium ions are recognized to be integral components of normal metabolic processes. However, some restrictions have been placed on levels of ammonium salts allowable in processed of foods. Maximum allowable levels in processed foods are as follows: $0.04-3.2 \%$ ammonium bicarbonate in baked goods, grain, snack, foods and reconstituted vegetables; $2.0 \%$ ammonium carbonate in baked goods, gelatins and puddings; $0.001 \%$ ammonium chloride in baked goods and $0.8 \%$ in condiments and relishes; $0.6-0.8 \%$ ammonium hydroxide in baked goods, cheeses, gelatins and puddings; $0.01 \%$ monobasic ammonium phosphate in baked goods; and $1.1 \%$ dibasic ammonium phosphate in baked goods, $0.003 \%$ in nonalcoholic beverages, and $0.012 \%$ for condiments and relishes. 
Table 8-1. Regulations and Guidelines Applicable to Ammonia

\begin{tabular}{|c|c|c|c|}
\hline Agency & Description & Information & References \\
\hline \multicolumn{4}{|c|}{ INTERNATIONAL } \\
\hline \multicolumn{4}{|c|}{ Guidelines: } \\
\hline IARC & Carcinogenicity classification & No data & \\
\hline \multirow[t]{4}{*}{ WHO } & $\begin{array}{l}\text { Drinking water quality guideline } \\
\text { Ammonia }\end{array}$ & & WHO 2002 \\
\hline & Threshold odor concentration & $1.5 \mathrm{mg} / \mathrm{L}$ & \\
\hline & Threshold taste concentration & $35 \mathrm{mg} / \mathrm{L}$ & \\
\hline & Health-based guideline & None proposed & \\
\hline \multicolumn{4}{|c|}{ NATIONAL } \\
\hline \multicolumn{4}{|c|}{$\begin{array}{l}\text { Regulations and Guidelines: } \\
\text { a. Air }\end{array}$} \\
\hline \multirow[t]{6}{*}{ ACGIH } & TLV (8-hour TWA) & & ACGIH 2001 \\
\hline & Ammonia & 25 ppm & \\
\hline & Ammonium chloride fume & $10 \mathrm{mg} / \mathrm{m}^{3}$ & \\
\hline & STEL (15-minute TWA) & & ACGIH 2001 \\
\hline & Ammonia & 35 ppm & \\
\hline & Ammonia chloride fume & $20 \mathrm{mg} / \mathrm{m}^{3}$ & \\
\hline \multirow[t]{7}{*}{ EPA } & $\begin{array}{l}\text { Accidental release prevention; } \\
\text { toxic endpoint }\end{array}$ & & EPA 2002b \\
\hline & Ammonia (anhydrous) & $0.14 \mathrm{mg} / \mathrm{L}$ & $\begin{array}{l}\text { 40CFR68, } \\
\text { Appendix A }\end{array}$ \\
\hline & Ammonia ( $>20 \%$ concentration) & $0.14 \mathrm{mg} / \mathrm{L}$ & \\
\hline & $\begin{array}{l}\text { Regulated toxic substance for } \\
\text { accidental release prevention } \\
\text { under Section 112(r) of the Clean } \\
\text { Air Act; threshold quantity }\end{array}$ & & EPA 2002a \\
\hline & Ammonia (anhydrous) & 10,000 pounds & $\begin{array}{l}\text { 40CFR68.130, } \\
\text { Table } 1\end{array}$ \\
\hline & Ammonia ( $>20 \%$ concentration) & 20,000 pounds & \\
\hline & AEGL 1 (interim) & 30 ppm & EPA 2004 \\
\hline \multirow[t]{9}{*}{$\mathrm{NIOSH}$} & REL (10-hour TWA) & & NIOSH 2002b \\
\hline & Ammonia & 25 ppm & \\
\hline & Ammonium chloride fume & $10 \mathrm{mg} / \mathrm{m}^{3}$ & \\
\hline & STEL (15-minute TWA) & & NIOSH 2002b \\
\hline & Ammonia & 35 ppm & \\
\hline & Ammonium chloride fume & $20 \mathrm{mg} / \mathrm{m}^{3}$ & \\
\hline & IDLH & & NIOSH 2002b \\
\hline & Ammonia & 300 ppm & \\
\hline & Ammonium chloride fume & No data & \\
\hline \multirow[t]{2}{*}{ OSHA } & $\begin{array}{l}\text { PEL (8-hour TWA) for general } \\
\text { industry }\end{array}$ & & $\begin{array}{l}\text { OSHA 2002d } \\
\text { 29CFR1910.1000, } \\
\text { Table Z-1 }\end{array}$ \\
\hline & Ammonia & 50 ppm & \\
\hline
\end{tabular}


Table 8-1. Regulations and Guidelines Applicable to Ammonia

\begin{tabular}{|c|c|c|c|}
\hline Agency & Description & Information & References \\
\hline \multicolumn{4}{|c|}{ NATIONAL (cont.) } \\
\hline \multirow[t]{13}{*}{ OSHA } & $\begin{array}{l}\text { PEL (8-hour TWA) for construction } \\
\text { industry }\end{array}$ & & $\begin{array}{l}\text { OSHA 2002c } \\
\text { 29CFR1926.55, } \\
\text { Appendix A }\end{array}$ \\
\hline & Ammonia & 50 ppm & \\
\hline & $\begin{array}{l}\text { PEL (8-hour TWA) for shipyard } \\
\text { industry }\end{array}$ & & $\begin{array}{l}\text { OSHA 2002a } \\
\text { 29CFR1915.1000 }\end{array}$ \\
\hline & Ammonia & 50 ppm & \\
\hline & $\begin{array}{l}\text { Highly hazardous chemical, toxic, } \\
\text { and reactive for general industry; } \\
\text { threshold quantity }\end{array}$ & & $\begin{array}{l}\text { OSHA 2002e } \\
\text { 29CFR1910.119, } \\
\text { Appendix A }\end{array}$ \\
\hline & Ammonia & 10,000 pounds & \\
\hline & $\begin{array}{l}\text { Ammonia solutions ( }>44 \% \text { of } \\
\text { ammonia by weight) }\end{array}$ & 15,000 pounds & \\
\hline & $\begin{array}{l}\text { Highly hazardous chemical, toxic, } \\
\text { and reactive for construction } \\
\text { industry; threshold quantity }\end{array}$ & & $\begin{array}{l}\text { OSHA 2002f } \\
\text { 29CFR1926.64, } \\
\text { Appendix A }\end{array}$ \\
\hline & Ammonia & 10,000 pounds & \\
\hline & $\begin{array}{l}\text { Ammonia solutions ( }>44 \% \text { of } \\
\text { ammonia by weight) }\end{array}$ & 15,000 pounds & \\
\hline & $\begin{array}{l}\text { Occupational safety and health } \\
\text { standards; storage and handling } \\
\text { of anhydrous ammonia }\end{array}$ & & $\begin{array}{l}\text { OSHA } 2002 \mathrm{~g} \\
\text { 29CFR1910.111 }\end{array}$ \\
\hline & $\begin{array}{l}\text { Occupations involved in } \\
\text { agriculture that are particularly } \\
\text { hazardous for the employment of } \\
\text { children below the age of } 16\end{array}$ & $\begin{array}{l}\text { Transporting, } \\
\text { transferring, or applying } \\
\text { anhydrous ammonia }\end{array}$ & $\begin{array}{l}\text { OSHA } 1998 \\
\text { 29CFR570.71(a)(11) }\end{array}$ \\
\hline & $\begin{array}{l}\text { Safety and health regulations for } \\
\text { construction; blasting and use of } \\
\text { explosives; common blasting } \\
\text { agent is a mixture of ammonium } \\
\text { nitrate and carbonaceous } \\
\text { combustibles }\end{array}$ & & $\begin{array}{l}\text { OSHA 2002b } \\
\text { 29CFR1926.914(e) }\end{array}$ \\
\hline \multicolumn{4}{|l|}{ b. Water } \\
\hline EPA & $\begin{array}{l}\text { Hazardous substance designated } \\
\text { pursuant to Section } 311(\mathrm{~b})(2)(\mathrm{A}) \\
\text { of the Clean Water Act } \\
\text { Ammonia } \\
\text { Ammonium chloride } \\
\text { Ammonium fluoride } \\
\text { Ammonium hydroxide }\end{array}$ & & $\begin{array}{l}\text { EPA } 2002 \mathrm{~h} \\
\text { 40CFR116.4, } \\
\text { Table A }\end{array}$ \\
\hline \multirow[t]{5}{*}{ EPA } & $\begin{array}{l}\text { Reportable quantity of hazardous } \\
\text { substances designated pursuant } \\
\text { to Section } 311 \text { of the Clean Water } \\
\text { Act }\end{array}$ & & $\begin{array}{l}\text { EPA 2002i } \\
\text { 40CFR117.3 }\end{array}$ \\
\hline & Ammonia & 100 pounds & \\
\hline & Ammonium chloride & 5,000 pounds & \\
\hline & Ammonium fluoride & 100 pounds & \\
\hline & Ammonium hydroxide & 1,000 pounds & \\
\hline
\end{tabular}


Table 8-1. Regulations and Guidelines Applicable to Ammonia

\begin{tabular}{|c|c|c|c|}
\hline Agency & Description & Information & References \\
\hline \multicolumn{4}{|c|}{ NATIONAL (cont.) } \\
\hline USC & $\begin{array}{l}\text { Assurances of availability of } \\
\text { adequate supplies of chemicals } \\
\text { necessary for treatment of water }\end{array}$ & Ammonia & $\begin{array}{l}\text { USC 2002a } \\
42 \text { USC } 300 j\end{array}$ \\
\hline \multicolumn{4}{|l|}{ c. Food } \\
\hline \multirow[t]{3}{*}{ EPA } & $\begin{array}{l}\text { Residues from ammonium } \\
\text { chloride, ammonium hydroxide, } \\
\text { and ammonium sulfate are } \\
\text { exempted from the requirement of } \\
\text { a tolerance when used in } \\
\text { accordance with good agricultural } \\
\text { practice as inert (or occasionally } \\
\text { inactive) ingredients in pesticide } \\
\text { formulations applied to growing } \\
\text { crops or to raw agricultural } \\
\text { commodities after harvest }\end{array}$ & & $\begin{array}{l}\text { EPA 2002e } \\
\text { 40CFR180.1001(c) }\end{array}$ \\
\hline & $\begin{array}{l}\text { Ammonium nitrate is exempt from } \\
\text { the requirement of a tolerance } \\
\text { when used in accordance with } \\
\text { good agricultural practice as inert } \\
\text { (or occasionally active) ingredients } \\
\text { in pesticide formulations applied to } \\
\text { growing crops only }\end{array}$ & & $\begin{array}{l}\text { EPA 2002e } \\
\text { 40CFR180.1001(d) }\end{array}$ \\
\hline & $\begin{array}{l}\text { The fungicide ammonia is } \\
\text { exempted from the requirement of } \\
\text { a tolerance when used after } \\
\text { harvest on the raw agricultural } \\
\text { commodities grapefruit, lemons, } \\
\text { oranges, and corn grain for feed } \\
\text { use only }\end{array}$ & & $\begin{array}{l}\text { EPA } 2002 f \\
\text { 40CFR180.1003 }\end{array}$ \\
\hline \multirow[t]{2}{*}{ FDA } & $\begin{array}{l}\text { Direct food substances affirmed } \\
\text { as generally recognized as safe }\end{array}$ & Ammonium chloride & $\begin{array}{l}\text { FDA 2001a } \\
\text { 21CFR184.1138 }\end{array}$ \\
\hline & $\begin{array}{l}\text { Direct food substances affirmed } \\
\text { as generally recognized as safe }\end{array}$ & Ammonium hydroxide & $\begin{array}{l}\text { FDA 2001b } \\
\text { 21CFR184.1139 }\end{array}$ \\
\hline \multirow[t]{7}{*}{ FDA } & $\begin{array}{l}\text { Direct food substances affirmed } \\
\text { as generally recognized as safe }\end{array}$ & Ammonium sulfate & $\begin{array}{l}\text { FDA 2001c } \\
\text { 21CFR184.1143 }\end{array}$ \\
\hline & $\begin{array}{l}\text { Drug products containing certain } \\
\text { active ingredients offered over- } \\
\text { the-counter }\end{array}$ & Ammonium chloride & $\begin{array}{l}\text { FDA 2001d } \\
\text { 21CFR310.545(a) }\end{array}$ \\
\hline & Expectorant drug product & Ammonia solution & \\
\hline & $\begin{array}{l}\text { Fever blister and cold sore } \\
\text { treatment drug product }\end{array}$ & Ammonia solution and & \\
\hline & Insect bite and sting drug products & Ammonium hydroxide & \\
\hline & $\begin{array}{l}\text { Food additives permitted in feed } \\
\text { and drinking water of animals }\end{array}$ & Anhydrous ammonia & $\begin{array}{l}\text { FDA 2001e } \\
\text { 21CFR573.180 }\end{array}$ \\
\hline & $\begin{array}{l}\text { Substance generally recognized } \\
\text { as safe when used in accordance } \\
\text { with good manufacturing or } \\
\text { feeding practices }\end{array}$ & Ammonium hydroxide & $\begin{array}{l}\text { FDA } 2001 \mathrm{f} \\
\text { 21CFR582.1139 }\end{array}$ \\
\hline
\end{tabular}


Table 8-1. Regulations and Guidelines Applicable to Ammonia

\begin{tabular}{|c|c|c|c|}
\hline Agency & Description & Information & References \\
\hline \multicolumn{4}{|l|}{ NATIONAL (cont.) } \\
\hline & $\begin{array}{l}\text { Substance generally recognized } \\
\text { as safe when used in accordance } \\
\text { with good manufacturing or } \\
\text { feeding practices }\end{array}$ & Ammonium sulfate & $\begin{array}{l}\text { FDA } 2001 \mathrm{~g} \\
\text { 21CFR582.1143 }\end{array}$ \\
\hline \multicolumn{4}{|l|}{ d. Other } \\
\hline \multirow[t]{2}{*}{ CPSC } & Federal Caustic Poison Act & & CPSC 2001 \\
\hline & $\begin{array}{l}\text { Ammonia water and any } \\
\text { preparation containing free or } \\
\text { chemically uncombined } \\
\text { ammonia, including ammonium } \\
\text { hydroxide and "hartshorn", in a } \\
\text { concentration of } 5 \% \text { or more }\end{array}$ & & 16CFR1500.129(1) \\
\hline \multirow[t]{10}{*}{ EPA } & Ammonia & & IRIS 2004 \\
\hline & Carcinogenicity classification & No data & \\
\hline & $\mathrm{RfC}$ & $1 \times 10^{-1} \mathrm{mg} / \mathrm{m}^{3}$ & \\
\hline & RfD & No data & \\
\hline & $\begin{array}{l}\text { CERCLA hazardous substance } \\
\text { designated pursuant to } \\
\text { Section } 311(b)(4) \text { of the Clean } \\
\text { Water Act }\end{array}$ & & EPA 2002d \\
\hline & Reportable quantity & & 40CFR302.4(a) \\
\hline & Ammonia & 100 pounds & \\
\hline & Ammonium chloride & 5,000 pounds & \\
\hline & Ammonium fluoride & 100 pounds & \\
\hline & Ammonium hydroxide & 1,000 pounds & \\
\hline \multirow[t]{7}{*}{ EPA } & Extremely hazardous substance & & EPA 2002c \\
\hline & Ammonia & & 40CFR355, \\
\hline & Reportable quantity & 100 pounds & Appendix A \\
\hline & Threshold planning quantity & 500 pounds & \\
\hline & $\begin{array}{l}\text { Toxic chemical release reporting; } \\
\text { Community right-to-know; } \\
\text { effective date for reporting }\end{array}$ & & EPA 2002g \\
\hline & Ammonia $^{\mathrm{b}}$ & $01 / 01 / 87$ & 40CFR372.65(a) \\
\hline & Ammonium nitrate (solution) & $01 / 01 / 87^{c}$ & \\
\hline \multirow[t]{4}{*}{ USC } & $\begin{array}{l}\text { Imposition of Superfund tax on } \\
\text { any taxable chemical sold by the } \\
\text { manufacturer, producer, or } \\
\text { importer }\end{array}$ & & USC 2002d \\
\hline & Ammonia & $\$ 2.64$ per ton & 26USC4661 \\
\hline & $\begin{array}{l}\text { Refund or credit of Superfund tax } \\
\text { paid when ammonia is used as a } \\
\text { fertilizer }\end{array}$ & & $\begin{array}{l}\text { USC } 2002 \mathrm{~b} \\
\text { 26USC4662 }\end{array}$ \\
\hline & Superfund taxable substance & Ammonium nitrate & $\begin{array}{l}\text { USC 2002c } \\
26 U S C 4672\end{array}$ \\
\hline
\end{tabular}

\section{STATE}

Regulations and Guidelines:

a. Air

No data 
Table 8-1. Regulations and Guidelines Applicable to Ammonia

\begin{tabular}{|c|c|c|c|}
\hline Agency & Description & Information & References \\
\hline \multicolumn{4}{|l|}{ STATE (cont.) } \\
\hline b. Water & & No data & \\
\hline c. Food & & No data & \\
\hline d. Other & & No data & \\
\hline Florida & $\begin{array}{l}\text { Toxic substance } \\
\text { Ammonia } \\
\text { Ammonium chloride } \\
\text { Ammonium fluoride } \\
\text { Ammonium nitrate } \\
\text { Ammonium sulfate }\end{array}$ & & BLR 2002 \\
\hline Massachusetts & $\begin{array}{l}\text { Hazardous substance } \\
\text { Ammonia } \\
\text { Ammonium chloride } \\
\text { Ammonium fluoride } \\
\text { Ammonium hydroxide } \\
\text { Ammonium nitrate } \\
\text { Ammonium sulfate }\end{array}$ & & BLR 2002 \\
\hline Minnesota & $\begin{array}{l}\text { Hazardous substance } \\
\text { Ammonia } \\
\text { Ammonium chloride, fume }\end{array}$ & & BLR 2002 \\
\hline New Jersey & $\begin{array}{l}\text { Hazardous substance } \\
\text { Ammonia }\end{array}$ & & BLR 2002 \\
\hline New York & $\begin{array}{l}\text { Hazardous substance } \\
\text { Ammonia } \\
\text { Ammonium chloride } \\
\text { Ammonium fluoride } \\
\text { Ammonium hydroxide }\end{array}$ & & BLR 2002 \\
\hline Pennsylvania & $\begin{array}{l}\text { Hazardous substance } \\
\text { Ammonia } \\
\text { Ammonium chloride } \\
\text { Ammonium fluoride } \\
\text { Ammonium hydroxide }\end{array}$ & & BLR 2002 \\
\hline
\end{tabular}

\footnotetext{
${ }^{a}$ Potential for a catastrophic event at or above the threshold quantity.

${ }^{\mathrm{b}}$ Ammonia: includes anhydrous ammonia, aqueous ammonia from water, dissociable ammonium salts, and other sources; $10 \%$ of total aqueous ammonia is reportable under this listing.

${ }^{\mathrm{c}}$ Ammonium nitrate (solution) is removed from this listing; the removal is effective 07/02/95, for the 1995 reporting year.

ACGIH = American Conference of Governmental Industrial Hygienists; $A E G L=$ acute exposure guideline value: BLR = Business \& Legal Reports, Inc. CERCLA = Comprehensive Environmental Response, Compensation, and Liability Act; CFR = Code of Federal Regulations; CPSC = Consumer Protection Safety Commission;

EPA = Environmental Protection Agency; FDA = Food and Drug Administration; IARC = International Agency for Research on Cancer; IDLH = immediately dangerous to life and health; IRIS = Integrated Risk Information System; $\mathrm{NIOSH}=$ National Institute of Occupational Safety and Health; OSHA = Occupational Safety and Health Administration; PEL = permissible exposure limit; ppm = parts per million; REL = recommended exposure limit; $\mathrm{RfC}=$ inhalation reference concentration; $\mathrm{RfD}=$ oral reference dose; STEL = short-term exposure limit; TLV = threshold limit value; TWA = time-weighted average; USC = United States Code; WHO = World Health Organization
} 